\title{
Adaptive statistical parametric mapping for fMRI
}

\author{
Ping Bai ${ }^{\dagger}$, Haipeng Shen ${ }^{\ddagger}$, Jianhua Z. HuAng ${ }^{\S}$ And \\ Young K. TruOnG ${ }^{\dagger, *}$
}

Brain activity is accompanied by changes in cerebral blood flow (CBF) and the differential blood oxygenation that are detectable using functional magnetic resonance imaging (fMRI). The process of identifying brain activation regions can be facilitated by estimating the hemodynamic response function (HRF). There have been some remarkable new developments in statistics to handle this problem. In this paper, we introduce a novel procedure which is capable of adapting itself to any of the existing methods by improving its performance through the application of a penalized smoothing technique. Using a computer experiment and a real fMRI data set, the proposed procedure is assessed by comparing its performance very favorably to the popular SPM based method.

AMS 2000 SUbJeCt Classifications: Primary 62H35, 62G08; secondary $62 \mathrm{H} 12$.

\section{INTRODUCTION}

Functional magnetic resonance imaging (fMRI) is a noninvasive medical method for detecting the temporal hemodynamic response to a given stimulus. This is possible because of the differential magnetic susceptibility of oxygenated and deoxygenated hemoglobins. Thus the response is also referred to as the blood oxygenation level dependent (BOLD) effect. The data is described in terms of 3 dimensional grids of $M$ volume elements (voxels), which is scanned repeatedly over a period of time so that each voxel will possess (say) $N$ time point BOLD effects. In practice, we denote the BOLD effect of the $j$ th voxel recorded at time $i$ by $y_{i j}$, where $1 \leq i \leq N$ and $1 \leq j \leq M$. Here $M$ is a scanner resolution specific constant. Typically, $M \sim 10^{5}$ and $N \sim 10^{2}$. Based on this vast amount of data, one of the objectives of fMRI is to identify which voxel has responded to the experimental stimulus.

Model-driven strategies have been playing a dominant role in relating the stimulus to the hemodynamic response. Among them, the most widely used approach is based on the general linear model (GLM) given by

$$
\mathbf{Y}=\mathbf{X} \boldsymbol{\beta}+\boldsymbol{\epsilon},
$$

* Corresponding author.

${ }^{\dagger}$ Research partially supported by NSF DMS-0707090

${ }^{\ddagger}$ Research partially supported by NSF DMS-0606577

$\S$ Research partially supported by NSF DMS-0606580

where $\mathbf{Y}=\left(y_{i j}\right)_{N \times M}$ is the BOLD effect data, $\mathbf{X}=$ $\left(x_{i k}\right)_{N \times(K+1)}$ is related to the stimuli, $\boldsymbol{\beta}=\left(\beta_{k j}\right)_{(K+1) \times M}$ is a matrix of parameters, and $\boldsymbol{\epsilon}=\left(\epsilon_{i j}\right)_{N \times M}$ whose columns consist of noise series with mean zero and covariance $\sigma^{2} \boldsymbol{\Sigma}$. Suppose there are $K$ different conditions involved in the experiment. For example, these conditions correspond to four different hand movements included in one of our experiments of the fMRI study [15]. Then $\mathbf{X}$ is an $N \times(K+1)$ design matrix, where the first column is usually constant representing the condition when the subject is at rest with no experimental activities performed, and each of the remaining columns contains the time component for an experiment condition. The $(K+1) \times M$ matrix $\boldsymbol{\beta}$ contains the related parameters, and due to the special formulation of the design matrix, the second to the last rows naturally give the contrast between each experiment condition and the benchmark when the subject is at rest. The inferences on each row of $\boldsymbol{\beta}$ will generate a spatial map related to one experiment condition.

The construction of the spatial map is carried out in two steps. In step one, a time series regression method is carried out at each voxel in order to obtain the estimate of the effect of the stimulus on the BOLD signals. That is, we regress each voxel time series $\mathbf{Y}_{j}$ (a column vector of $\mathbf{Y}$ ) on $\mathbf{X}$ via

$$
\mathbf{Y}_{j}=\mathbf{X} \boldsymbol{\beta}_{j}+\boldsymbol{\epsilon}_{j}, \quad \boldsymbol{\epsilon}_{j} \sim\left(\mathbf{0}, \sigma^{2} \boldsymbol{\Sigma}\right), \quad j=1,2, \ldots, M .
$$

Here $\boldsymbol{\beta}_{j}$ is the $j$ th column of $\boldsymbol{\beta}$. Once this model has been fitted at each voxel, inferences of the model parameters are then made according to the experiment hypothesis. The resulting statistics from all the voxels are assembled spatially into an image, which is the so-called statistical parametric map. The second step then focuses on the analysis of the statistical map in order to identify those areas of the brain that are activated by the stimuli [4]. For more detailed discussions, see statistical parametric mapping (SPM [3]), FMRIB Software Library (FSL [16]) and AFNI [2].

The nature of the BOLD response implies that in areas of activation there is a delayed and blurred version of the stimulus sequence (Figure 1). Hence each column of the design matrix $\mathbf{X}$, which represents the temporal characteristic of the expected response, is commonly modeled through the 

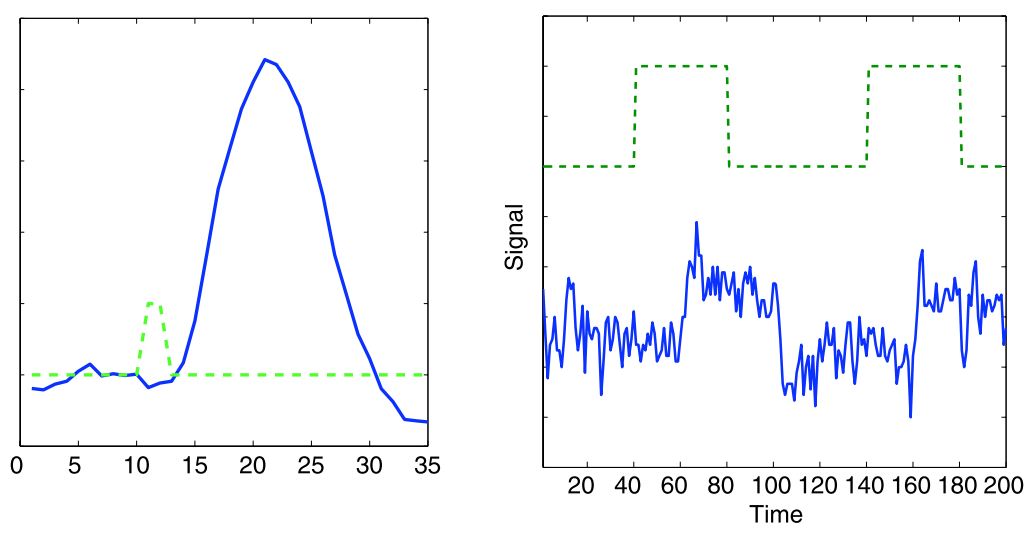

Figure 1. Left Panel: The recorded BOLD signal (solid line) triggered by a single event (dashed line). Right Panel: The recorded BOLD signal (solid line) triggered by a typical block-design sequence (dashed line).
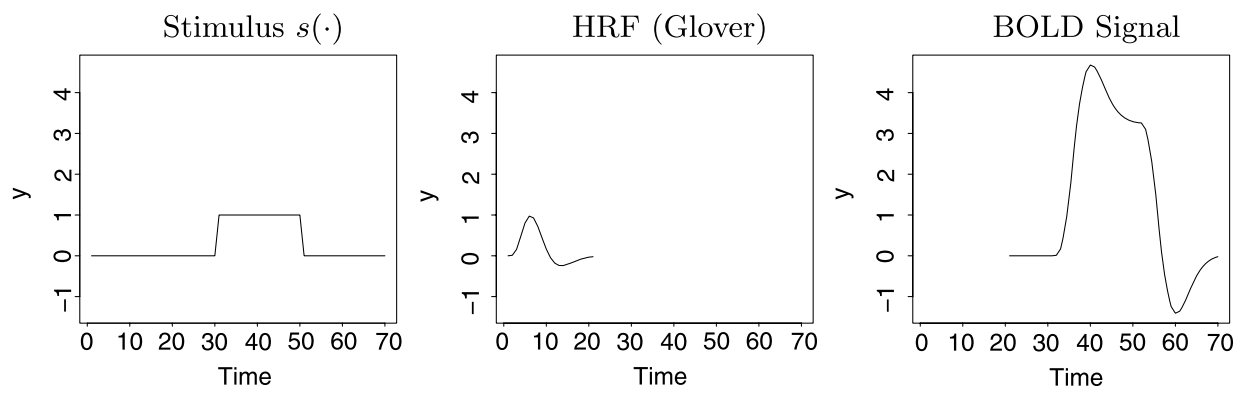

Figure 2. A block design stimulus $s(\cdot)$ (left). A model of the hemodynamic response function $h(\cdot)$ for motor response [6] (middle), and the BOLD response $y(t)=s \otimes h(t)=\sum_{u} h(t-u) s(u)$ (right).

convolution of the stimulus design $s(\cdot)$ with a hemodynamic response function $(\mathrm{HRF}), h(\cdot)$. That is,

$$
\mathbf{X}_{k}(t)=\sum_{u} h(t-u) s_{k}(u),
$$

where $\mathbf{X}_{k}$ is the $k$ th column of the design matrix. $s_{k}(\cdot)$ is the stimulus sequence of the $k$ th experiment condition, which usually consists of zeros and ones, where one stands for activation and zero stands for rest.

The modeled $\mathbf{X}_{k}$ then indicates the temporal characteristic of the brain regions that are activated by the $k$ th experiment condition. In the literature, commonly used forms of HRF include discretized Poisson, Gamma, Gaussian density functions and more realistically the two-gamma model [6] (middle of Figure 2). However, these HRFs assume predefined parametric forms, which are rather restrictive. Some work has been proposed to model the HRF using a small set of temporal basis functions to improve the flexibility of its form $[4 ; 10]$. One drawback of the basis approach is that it introduces less sensitivity of the estimation and the results are more difficult to interpret [11].

There have been many new developments in the estimation of HRF over the last few years. There is spatio-temporal regularization method [17], nonparametric methods such as those based on orthonormal causal Laguerre polynomials [14], wavelet approach [12], spline methods [5; 19; 18], and the frequency domain coherence based method [1]. There are also data-driven and model-driven methods [9; 13]. Instead of assuming a certain form for the HRF, the authors model the HRF or the temporal component directly making use of the information extracted by some data-driven methods, such as independent component analysis (ICA) or principal component analysis (PCA).

In this work, we propose a new nonparametric method to model the time component adaptively in the context of SPM. The idea is to start from an initial design matrix $\mathbf{X}$ computed from a procedure capable of modeling the HRF such as SPM. Then invoke a penalized smoothing technique to update the estimate of the hemodynamic response iteratively. This is called an adaptive procedure because it uses the timing information of various experimental stimuli offered by the experiment design, and adjusts the shape of the temporal response of the activated voxels so that it is closer to the reality. Because of the improved accuracy of the estimated temporal characteristic, the corresponding detection power of the activated regions is increased as well. The performance of the proposed method is illustrated through a simulation study, as well as a comparative study on a real fMRI data. 
The rest of this paper is organized as follows. The description of our proposed method is given in Section 2. Section 3 reports a simulation study to illustrate its performance. A comparative study on a real fMRI data set is presented in Section 4. Some discussion is given in the final section.

\section{ADAPTIVE SPM}

The GLM model (1.1) can also be expressed as

$$
\begin{aligned}
y_{i j}=x_{i 0} \beta_{0 j}+x_{i 1} \beta_{1 j}+\cdots+ & x_{i K} \beta_{K j}+\epsilon_{i j}, \\
& i=1, \ldots, N, j=1, \ldots, M .
\end{aligned}
$$

Hence, for the image recorded at time $i=1, \ldots, N$, we have

$$
\begin{aligned}
\mathbf{Y}_{(i)}= & \left(y_{i 1}, \ldots, y_{i M}\right)^{T} \\
= & x_{i 0}\left(\beta_{01}, \ldots, \beta_{0 M}\right)^{T}+x_{i 1}\left(\beta_{11}, \ldots, \beta_{1 M}\right)^{T}+\cdots+ \\
& \quad+x_{i K}\left(\beta_{K 1}, \ldots, \beta_{K M}\right)^{T}+\boldsymbol{\epsilon}_{(i)} \\
& =x_{i 0} \boldsymbol{\beta}_{(0)}+x_{i 1} \boldsymbol{\beta}_{(1)}+\cdots+x_{i K} \boldsymbol{\beta}_{(K)}+\boldsymbol{\epsilon}_{(i)},
\end{aligned}
$$

where $\boldsymbol{\epsilon}_{(i)}=\left(\epsilon_{i 1}, \epsilon_{i 2}, \ldots, \epsilon_{i M}\right)^{T}, \boldsymbol{\beta}_{(k)}=\left(\beta_{k 1}, \ldots, \beta_{k M}\right)^{T}$ for $k=0,1, \ldots, K$. Concatenating all the images recorded at different time points, we have

$$
\begin{aligned}
& \left(\begin{array}{c}
\mathbf{Y}_{(1)} \\
\mathbf{Y}_{(2)} \\
\vdots \\
\mathbf{Y}_{(N)}
\end{array}\right)=\left(\begin{array}{cccc}
\boldsymbol{\beta}_{(0)} & \tilde{0} & \cdots & \tilde{0} \\
\tilde{0} & \boldsymbol{\beta}_{(0)} & \cdots & \tilde{0} \\
\vdots & \vdots & \ddots & \vdots \\
\tilde{0} & \tilde{0} & \cdots & \boldsymbol{\beta}_{(0)}
\end{array}\right) \mathbf{X}_{0}+\cdots+ \\
& +\left(\begin{array}{cccc}
\boldsymbol{\beta}_{(K)} & \tilde{0} & \cdots & \tilde{0} \\
0 & \boldsymbol{\beta}_{(K)} & \cdots & \tilde{0} \\
\vdots & \vdots & \ddots & \vdots \\
\tilde{0} & \tilde{0} & \cdots & \boldsymbol{\beta}_{(K)}
\end{array}\right) \mathbf{X}_{K}+\left(\begin{array}{c}
\boldsymbol{\epsilon}_{(1)} \\
\boldsymbol{\epsilon}_{(2)} \\
\vdots \\
\boldsymbol{\epsilon}_{(N)}
\end{array}\right),
\end{aligned}
$$

where $\mathbf{X}_{k}=\left(x_{1 k}, \ldots, x_{N k}\right)^{T}$, for $k=0,1, \ldots, K$ and $\tilde{0}$ is an $M \times 1$ vector of zeros.

SPM fits the above GLM by assuming $\mathbf{X}_{0}=$ $(1,1, \ldots, 1)^{T}$, and obtaining $\mathbf{X}_{1}, \ldots, \mathbf{X}_{K}$ by convoluting a predefined HRF with the experimental stimulus sequence for each condition, respectively. For example, the canonical HRF used in SPM5 is a typical BOLD impulse response characterized by two gamma functions, one modeling the peak and the other modeling the undershoot. The canonical HRF is plotted in the middle of Figure 2.

By convoluting the canonical HRF with the stimulus sequence of each condition in the experiment, we obtain an initial design matrix $\mathbf{X}$. To make Model (2.1) identifiable, we assume $\left\|\mathbf{X}_{0}\right\|=\left\|\mathbf{X}_{1}\right\|=\cdots=\left\|\mathbf{X}_{K}\right\|=1$. In the GLM analysis of fMRI, the stimulus sequence is usually represented by a sequence of zeros and ones, where zero means rest and one means activation by a certain condition. Hence, after convoluting with the selected HRF, the modeled time component for a certain condition consists of nonzero values only during the activation period. Figure 2 illustrates this process. The plot on the leftmost shows a binary stimulus sequence $(1=$ activation, $0=$ rest). The plot in the middle is the canonical HRF [6] and the temporal component modeled through the convolution is plotted in the rightmost graph. We can obtain the starting and ending points of cerebral blood flow for each experiment stimulus from the initial design matrix (since the stimulus and the canonical HRF are both known). Our goal is then to estimate the shape of the nonzero part of the blood flow nonparametrically.

We propose to estimate the temporal and spatial components $(\mathbf{X}$ and $\boldsymbol{\beta})$ in an iterative way as described in the algorithm below. Each step is then elaborated on in sections that follow.

\section{Adaptive SPM Algorithm}

(1) Obtain the initial design matrix $\mathbf{X}$ from SPM5.

(2) Alternate until the convergence of $\mathbf{X}$ and $\boldsymbol{\beta}$.

(a) Based on the given $\mathbf{X}$, solve a GLM equation $\mathbf{Y}=$ $\mathbf{X} \boldsymbol{\beta}+\boldsymbol{\epsilon}$ to obtain $\boldsymbol{\beta}$

(b) Once $\boldsymbol{\beta}$ is obtained, use a penalization procedure to derive $X_{1}, \ldots, X_{K}$ smoothly.

\subsection{Obtain the initial design matrix}

At the beginning of the algorithm, the initial design matrix $\mathbf{X}$ is obtained from, for example, SPM5. The first column of $\mathbf{X}$ is a vector of ones. Each of the remaining columns is obtained by convoluting the stimulus sequence of a certain condition with a canonical HRF [6]. Each stimulus sequence consists of ones and zeros, where one means activation and zero means rest. Due to the multiplicative structure between $\mathbf{X}$ and $\boldsymbol{\beta}$ in Model (2.1), a scale factor can be transferred between them, which leads to scale ambiguity. To avoid that, we standardize each column of $\mathbf{X}$ to have norm 1 .

\subsection{Obtain the spatial map}

Based on the obtained design matrix $\mathbf{X}$, we can solve (2.1) in the form of $\mathbf{Y}=\mathbf{X} \boldsymbol{\beta}+\boldsymbol{\epsilon}, \boldsymbol{\epsilon} \sim\left(0, \sigma^{2} \boldsymbol{\Sigma}\right)$, where $\mathbf{X}=\left(\mathbf{X}_{0}, \mathbf{X}_{1}, \ldots, \mathbf{X}_{K}\right)$ and $\boldsymbol{\beta}=\left(\boldsymbol{\beta}_{(0)}^{T}, \boldsymbol{\beta}_{(1)}^{T}, \ldots, \boldsymbol{\beta}_{(K)}^{T}\right)^{T}$. For example, assuming the constant variance and independent noise structure, the ordinary least squares estimate of $\boldsymbol{\beta}$ is given by $\hat{\boldsymbol{\beta}}=\left(\mathbf{X}^{T} \mathbf{X}\right)^{-1} \mathbf{X}^{T} \mathbf{Y}$.

\subsection{Obtain the smooth time components}

After $\hat{\boldsymbol{\beta}}$ is obtained, we can then use the following penalization procedure to derive $\mathbf{X}_{1}, \ldots, \mathbf{X}_{K}$ smoothly. Note that $\mathbf{X}_{0}$ remains fixed because it stands for the background rest activity.

SPM assumes the same HRF for all the experimental conditions, hence it is reasonable to assume that their time components share the same nonzero part. (This works well for our current fMRI studies where the durations of the stimuli are all equal [15]. It is important to assess the performance 
of our method in experiments implemented with different durations.) However the positions of the nonzero part vary among different time components. Denote the nonzero part of $\mathbf{X}_{k}, k=1, \ldots, K$, as $\widetilde{\mathbf{X}}$ and its length as $\tilde{N}$. Suppose the nonzero part of $\mathbf{X}_{k}$ starts at index $s_{k}$ and ends at index $e_{k}$.

Then Model (2.1) can be simplified to

$$
\begin{aligned}
\left(\begin{array}{c}
\mathbf{Y}_{(1)} \\
\mathbf{Y}_{(2)} \\
\vdots \\
\mathbf{Y}_{(N)}
\end{array}\right)= & \left(\begin{array}{cccc}
\boldsymbol{\beta}_{(0)} & \tilde{0} & \cdots & \tilde{0} \\
\tilde{0} & \boldsymbol{\beta}_{(0)} & \cdots & \tilde{0} \\
\vdots & \vdots & \ddots & \vdots \\
\tilde{0} & \tilde{0} & \cdots & \boldsymbol{\beta}_{(0)}
\end{array}\right) \mathbf{X}_{0}+ \\
& +\cdots+\sum_{k=1}^{K}\left(\begin{array}{ccc}
\tilde{0}_{s k} & \cdots & \tilde{0}_{s k} \\
\boldsymbol{\beta}_{(k)} & \cdots & \tilde{0} \\
\vdots & \ddots & \vdots \\
\tilde{0} & \cdots & \boldsymbol{\beta}_{(k)} \\
\tilde{0}_{e k} & \cdots & \tilde{0}_{e k}
\end{array}\right) \tilde{\mathbf{X}}+\boldsymbol{\epsilon},
\end{aligned}
$$

where $\tilde{0}_{s k}$ is a column vector of zeros with length $\left(s_{k}-1\right) \times M$ and $\tilde{0}_{e k}$ is a column vector of zeros with length $\left(N-e_{k}\right) \times M$.

Note that in the above model, only $\widetilde{\mathbf{X}}$ is unknown and needs to be estimated. Hence it makes sense to re-express the model in the following way,

$$
\tilde{\mathbf{Y}}=\left(\begin{array}{c}
\tilde{\mathbf{Y}}_{(1)} \\
\tilde{\mathbf{Y}}_{(2)} \\
\vdots \\
\tilde{\mathbf{Y}}_{(N)}
\end{array}\right)=\left(\begin{array}{c}
\tilde{\boldsymbol{\beta}}_{1} \\
\tilde{\boldsymbol{\beta}}_{2} \\
\vdots \\
\tilde{\boldsymbol{\beta}}_{N}
\end{array}\right) \tilde{\mathbf{X}}+\boldsymbol{\epsilon}
$$

where $\tilde{\mathbf{Y}}_{(i)}=\mathbf{Y}_{(i)}-\boldsymbol{\beta}_{(0)} \mathbf{X}_{0}$ and $\tilde{\boldsymbol{\beta}}_{i}$ is a matrix with dimension $M \times \tilde{N}$ that can be obtained from the known $\boldsymbol{\beta}_{(k)}$ 's, $i=1, \ldots, N, k=1, \ldots, K$.

To obtain a smooth $\widetilde{\mathbf{X}}$, we consider the following penalized least square (PLS) criterion:

$$
\begin{aligned}
\|\tilde{\mathbf{Y}}-\tilde{\boldsymbol{\beta}} \widetilde{\mathbf{X}}\|^{2}+\lambda \widetilde{\mathbf{X}}^{T} \boldsymbol{\Omega} \widetilde{\mathbf{X}} \\
\quad=\sum_{i=1}^{N}\left\|\tilde{\mathbf{Y}}_{i}-\tilde{\boldsymbol{\beta}}_{i} \widetilde{\mathbf{X}}\right\|^{2}+\lambda \widetilde{\mathbf{X}}^{T} \boldsymbol{\Omega} \widetilde{\mathbf{X}} \\
=\sum_{i=1}^{N}\left(\tilde{\mathbf{Y}}_{i}^{T} \tilde{\mathbf{Y}}_{i}-2 \tilde{\mathbf{Y}}_{i}^{T} \tilde{\boldsymbol{\beta}}_{i} \widetilde{\mathbf{X}}+\widetilde{\mathbf{X}}^{T} \tilde{\boldsymbol{\beta}}_{i}^{T} \tilde{\boldsymbol{\beta}}_{i} \widetilde{\mathbf{X}}\right)+\lambda \widetilde{\mathbf{X}}^{T} \boldsymbol{\Omega} \widetilde{\mathbf{X}}
\end{aligned}
$$

where $\tilde{\boldsymbol{\beta}}=\left(\tilde{\boldsymbol{\beta}}_{1}, \tilde{\boldsymbol{\beta}}_{2}, \ldots, \tilde{\boldsymbol{\beta}}_{N}\right)^{T}$. The above criterion involves two terms. The first term measures the goodness of fit of the GLM, while the second term penalizes the roughness of $\widetilde{\mathbf{X}}$. $\lambda$ is the smoothing parameter that optimally balances the two terms and $\boldsymbol{\Omega}$ is the $\tilde{N} \times \tilde{N}$ matrix that generates the smoothing penalty.

The minimizer of the above PLS criterion is

$$
\widehat{\widetilde{\mathbf{X}}}=\left(\sum_{i=1}^{N} \tilde{\boldsymbol{\beta}}_{i}^{T} \tilde{\boldsymbol{\beta}}_{i}+\lambda \boldsymbol{\Omega}\right)^{-1}\left(\sum_{i=1}^{N} \tilde{\boldsymbol{\beta}}_{i}^{T} \tilde{\mathbf{Y}}_{i}\right) .
$$

Once we obtain $\widehat{\widetilde{\mathbf{X}}}$, we can normalize it and plug it back to the right positions of each temporal component to form an updated $\mathbf{X}$.

\subsection{Selection of the smoothing parameter}

One thing that we need to consider in obtaining the smooth temporal components is the selection of the smoothing parameter $\lambda$. We propose to select $\lambda$ using generalized cross validation (GCV) as follows.

After obtaining $\widehat{\widetilde{\mathbf{X}}}$, we have

$$
\begin{aligned}
\widehat{\tilde{\mathbf{Y}}}=\tilde{\boldsymbol{\beta}} \widehat{\widetilde{\mathbf{X}}} & =\tilde{\boldsymbol{\beta}}\left(\sum_{i=1}^{N} \tilde{\boldsymbol{\beta}}_{i}^{T} \tilde{\boldsymbol{\beta}}_{i}+\lambda \boldsymbol{\Omega}\right)^{-1}\left(\sum_{i=1}^{N} \tilde{\boldsymbol{\beta}}_{i} \tilde{\mathbf{Y}}_{i}\right) \\
& =\tilde{\boldsymbol{\beta}}\left(\tilde{\boldsymbol{\beta}}^{T} \tilde{\boldsymbol{\beta}}+\lambda \boldsymbol{\Omega}\right)^{-1} \tilde{\boldsymbol{\beta}}^{T} \tilde{\mathbf{Y}}=\mathbf{S} \tilde{\mathbf{Y}},
\end{aligned}
$$

where $\mathbf{S}:=\tilde{\boldsymbol{\beta}}\left(\tilde{\boldsymbol{\beta}}^{T} \tilde{\boldsymbol{\beta}}+\lambda \boldsymbol{\Omega}\right)^{-1} \tilde{\boldsymbol{\beta}}^{T}$. Define the GCV [8] by

$$
\begin{aligned}
& \operatorname{GCV}(\lambda) \\
& =\frac{\|\tilde{\hat{\mathbf{Y}}}-\tilde{\mathbf{Y}}\|^{2} /(M N)}{(1-\operatorname{tr}(\mathbf{S}) /(M N))^{2}} \\
& =\frac{\sum_{i=1}^{N}\left\|\tilde{\boldsymbol{\beta}}_{i}\left(\sum_{i=1}^{N} \tilde{\boldsymbol{\beta}}_{i}^{T} \tilde{\boldsymbol{\beta}}_{i}+\lambda \boldsymbol{\Omega}\right)^{-1}\left(\sum_{i=1}^{N} \tilde{\boldsymbol{\beta}}_{i}^{T} \tilde{\mathbf{Y}}_{i}\right)\right\|^{2} /(M N)}{(1-\operatorname{tr}(\mathbf{S}) /(M N))^{2}} .
\end{aligned}
$$

We choose the $\lambda$ that minimizes the GCV from a set of candidates. We can either choose $\lambda$ inside the iteration loop or outside the iteration loop.

\subsection{Remarks and implementation details}

\subsubsection{Initial selection of HRF}

Our algorithm appears to be insensitive to the shape of the initial $\widetilde{\mathbf{X}}$. All the information we need from the initial design matrix used in SPM is the starting and ending time points of the cerebral blood flow for each component. Once we have that, instead of using the design matrix assumed in SPM, we can replace the nonzero entries with vectors of ones; our algorithm converges to the same results, needing only a few more iterations.

\subsubsection{Calculation of $\operatorname{tr}(\mathbf{S})$}

The matrix $\mathbf{S}$ defined in Equation (2.3) has dimension $M N \times \tilde{N}$. Considering the huge size of typical fMRI data set, the calculation of $\mathbf{S}$ and $\operatorname{tr}(\mathbf{S})$ is rather time and memory consuming. However, in the implementation of our algorithm, we can use the simple fact that, for any $m \times n$ matrix $\mathbf{A}$ and $n \times m$ matrix $\mathbf{B}, \operatorname{tr}(\mathbf{A B})=\operatorname{tr}(\mathbf{B A})$. Hence,

$$
\operatorname{tr}(\mathbf{S})=\operatorname{tr}\left(\tilde{\boldsymbol{\beta}}\left(\tilde{\boldsymbol{\beta}}^{T} \tilde{\boldsymbol{\beta}}+\lambda \Omega\right)^{-1} \tilde{\boldsymbol{\beta}}^{T}\right)=\operatorname{tr}\left(\tilde{\boldsymbol{\beta}}^{T} \tilde{\boldsymbol{\beta}}\left(\tilde{\boldsymbol{\beta}}^{T} \tilde{\boldsymbol{\beta}}+\lambda \Omega\right)^{-1}\right) .
$$

The last term will be used to obtain the trace of $\mathbf{S}$ directly since both $\tilde{\boldsymbol{\beta}}^{T} \tilde{\boldsymbol{\beta}}$ and $\left.\left(\tilde{\boldsymbol{\beta}}^{T} \tilde{\boldsymbol{\beta}}+\lambda \Omega\right)^{-1}\right)$ have been calculated and stored previously. This will help to improve the performance of our numerical procedures in analyzing the vast amount of fMRI datasets. 


\subsubsection{Calculation of GCV}

Note that in Model (2.2), only the nonzero $\tilde{\boldsymbol{\beta}}_{i}$ 's, that is, the $i$ 's $(i=1,2, \ldots, N)$ that belong to at least one experiment task period, contribute to the model. Hence we can ignore those times where there are no activities and reindex the nonzero $\tilde{\boldsymbol{\beta}}_{i}$ 's as $\tilde{\boldsymbol{\beta}}_{1}, \tilde{\boldsymbol{\beta}}_{2}, \ldots, \tilde{\boldsymbol{\beta}}_{N^{*}}$, where $N^{*}$ is the number of time points that belong to at least one activation period. Model (2.2) then becomes

$$
\tilde{\mathbf{Y}}=\left(\begin{array}{c}
\tilde{\mathbf{Y}}_{(1)} \\
\tilde{\mathbf{Y}}_{(2)} \\
\vdots \\
\tilde{\mathbf{Y}}_{\left(N^{*}\right)}
\end{array}\right)=\left(\begin{array}{c}
\tilde{\boldsymbol{\beta}}_{1} \\
\tilde{\boldsymbol{\beta}}_{2} \\
\vdots \\
\tilde{\boldsymbol{\beta}}_{N^{*}}
\end{array}\right) \tilde{\mathbf{X}}+\boldsymbol{\epsilon}
$$

The remaining computations can be carried out based on this reduced model then. This is another way to make the algorithm more efficient in the implementation.

\section{A SIMULATION STUDY}

\subsection{Data description}

According to the GLM model (1.1), we simulated an $N \times$ $M$ fMRI data matrix $\mathbf{Y}$ by first simulating the $N \times(K+1)$ design matrix $\mathbf{X}$ and the $(K+1) \times M$ parameter matrix $\boldsymbol{\beta}$ separately. The data matrix $\mathbf{Y}$ was then obtained as $\mathbf{Y}=$ $\mathbf{X} \boldsymbol{\beta}+\boldsymbol{\epsilon}$, where $\boldsymbol{\epsilon}$ is a randomly generated noise matrix from a normal distribution with mean 0 and variance 0.4788 . The variance is estimated from the real fMRI study in Section 4.

In this study, we set $K=4, M=30 \times 30 \times 10$ and $N=120$. The simulated data can be explained as follows: there are 4 different tasks involved in the experiment and we want to detect which brain regions are related to the four tasks respectively. Each column of the design matrix $\mathbf{X}$ represents the cerebral blood changes triggered by a particular task. Each row of $\boldsymbol{\beta}$ consists of the parameters related to one experiment task, which form a spatial map that indicates the brain areas activated by the certain task. The brain image recorded at one time point consists of 10 slices and there are $30 \times 30$ voxels on each slice. The brain image is recorded 120 times, and hence each column of $\mathbf{X}$ is a time series of length 120 . We "simulated" the time components using a recorded cerebral blood flow from a real fMRI study (Section 4). The length of the blood flow triggered by the task spans 30 time points $(\tilde{N}=30)$. All the four experimental components share the same shape of the hemodynamic response. However, the nonzero response for each component starts at different times since, in our experiment, the subject performs the four tasks sequentially with some rest period in between.

All the voxels in $\boldsymbol{\beta}$ are given a numerical value of either 0 or 1 . In each spatial component, the voxels with value 1 correspond to the regions that are activated by the corresponding experiment task, and they are plotted as dark red areas in Figure 3 on page 38. The last time component is not fully observed in this experiment because the fMRI scan stopped right after the subject finished the last task. Due to the delay of the cerebral blood flow caused by hemodynamic response, we can only observe part of the blood flow in this case.

\subsection{Analysis and results}

We then applied our algorithm and SPM5 [3] to analyze the simulated data. As mentioned in the last section, there are four different conditions involved in the experiment $(K=4)$. Hence there are five columns in the design matrix $\mathbf{X}$, where the first column is a vector of 1 . The length of $\widetilde{\mathbf{X}}$, that is, the length of the hemodynamic triggered by the tasks, is set to be $30(\tilde{N}=30)$. The initial design matrix was obtained from SPM5. Namely, each column of $\mathbf{X}$, except for the first one, was obtained by convoluting the corresponding stimulus sequence with the canonical HRF (Figure 2 on page 34). Starting from this initial design matrix, our iterative algorithm converged after 10 iterations. The GCV criterion selected $\lambda$ to be $10^{3}$ from a set of candidate points of $\left\{0,10^{1}, 10^{2}, \ldots, 10^{10}\right\}$. We also applied the algorithm using a different initial design matrix, where $\widetilde{\mathbf{X}}$ is replaced by a vector of 1 . The algorithm converged to the same results after 15 iterations. The results are shown in the first column of Figure 4.

All the four components were recovered reasonably well, although some noise does exist due to the random noise added to the GLM model. Similar to Figure 3, dark red areas indicate the activated voxels. The time course plot in each panel shows the corresponding time component (solid lines). The estimated time components are very close to the real ones as in Figure 3.

The second column of Figure 4 shows the results using the design matrix obtained from SPM5. That is, we obtained the spatial maps by $\breve{\boldsymbol{\beta}}=\left(\breve{\mathbf{X}}^{T} \breve{\mathbf{X}}\right)^{-1} \breve{\mathbf{X}}^{T} \mathbf{Y}$, where $\breve{\mathbf{X}}$ is the design matrix used by SPM5. The time component modeled by the canonical HRF is shown in the last plot (solid line) in each panel. The shape of the time component is far away from the real ones. It's also obvious that the activation areas shown by the spatial maps are much more noisy than the activation indicated by our proposed method.

\section{A REAL DATA ANALYSIS}

\subsection{Experiment paradigm and data description}

To study brain regions that are related to different finger tapping movements, an fMRI data set was obtained from one human subject performing five different tasks alternately: rest, externally-guided (EG) right-hand movement, externally-guided left-hand movement, internallyguided (IG) right-hand movement and internally-guided left-hand movement. Each rest and activation period lasted 30 and 60 seconds, respectively. Here EG movement means 
Component 1
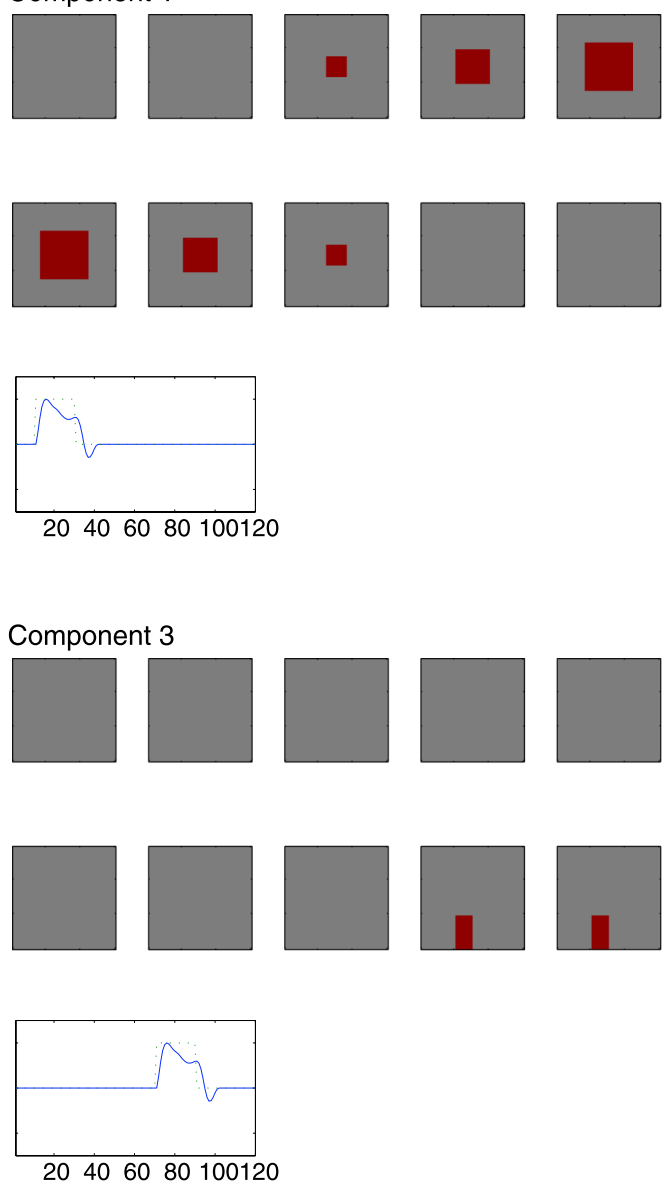

Component 2
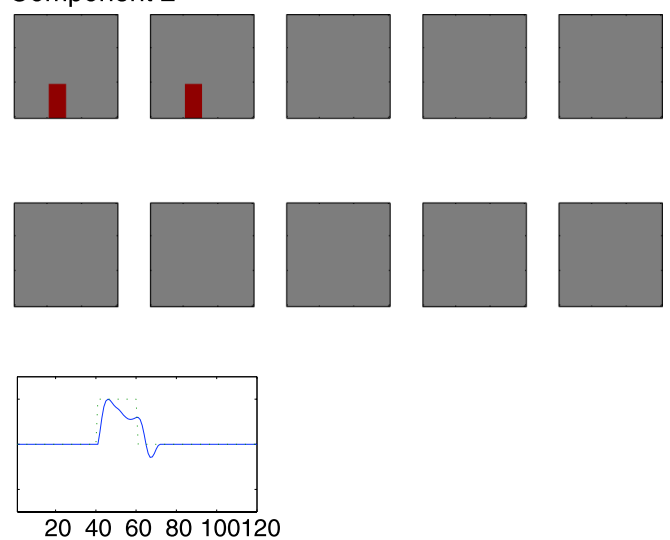

Component 4
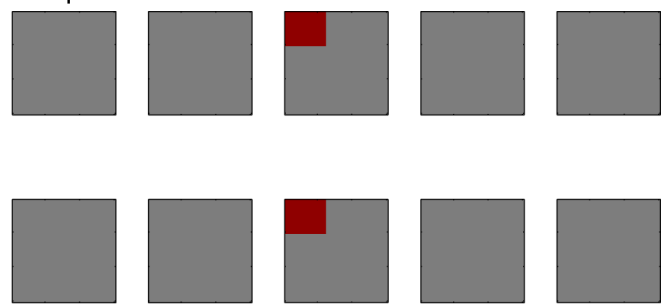

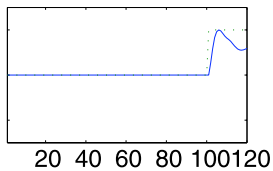

Figure 3. The four components used in the simulation. In each panel, the first 10 images are the spatial maps (one row of $\beta$ ), and the dark red areas stand for activated voxels. The solid line in the subsequent plot shows the temporal characteristic of the activated voxels (one column of $\mathbf{X}$ ). The dotted line stands for the experiment stimulus, 0 meaning "rest" and 1 meaning "active".

the subject did the sequential finger-tapping movements at $0.5 \mathrm{tap} / \mathrm{sec}$ following video instructions, while IG movement means the subject needed to finish the task according to their memory. The experimental paradigm is shown in Figure 5 . When acquiring the data, the subject repeated this paradigm sequence twice.

During the experiment, 240 magnetic resonance (MR) scans were acquired on a modified 3T Siemens MAGNETOM Vision system. Each acquisition consisted of 46 contiguous slices. Each slice contained $53 \times 63$ voxels. Hence there were $53 \times 63 \times 46$ voxels from each scan. The size of each voxel is $3 \mathrm{~mm} \times 3 \mathrm{~mm} \times 3 \mathrm{~mm}$. Each acquisition took 2.9388 seconds, with the scan to scan repetition time (TR) set to be 3 seconds.

\subsection{Analysis and results}

The goal of this fMRI study is to detect which brain regions are responsible for the four different finger tapping movements, EG right-hand (RE), IG right-hand (RI), EG left-hand (LE) and IG left-hand (LI).

The data set was preprocessed using SPM5, which included realignment, coregistration, segmentation, spatial normalization and smoothing. We then analyzed the preprocessed data set using both SPM5 with the canonical HRF and our proposed method with the initial design matrix obtained from SPM5.

The results from the two methods are displayed in the corresponding panels of Figure 6. As mentioned earlier, the goal of this study is to identify the brain regions activated by the four different finger movements. We only showed the four related brain slices for each movement according to the classic pattern mentioned in Section 4.1.

The upper panel of Figure 6 shows the four components detected by adaptive SPM. The lower panel presents the four components recovered by SPM. Within each row, the four image slices represent the activated spatial maps. The red areas illustrate activated brain regions. Brighter color 
Component 1
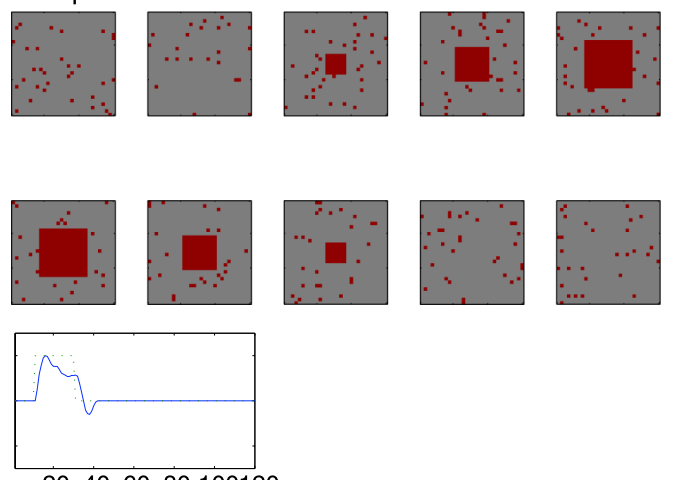

20406080100120

Component 2
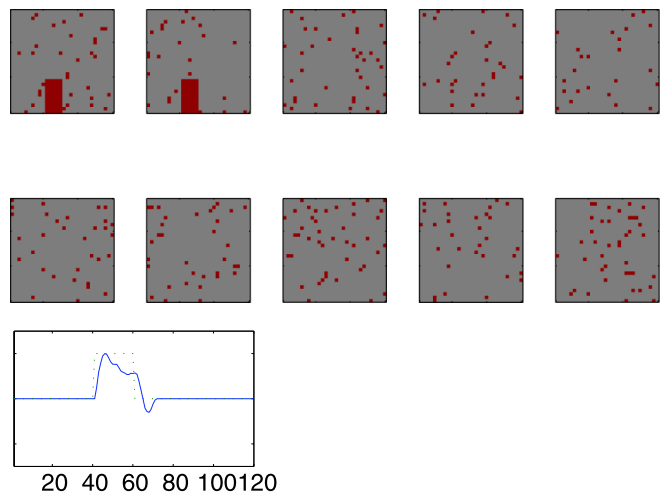

Component 3
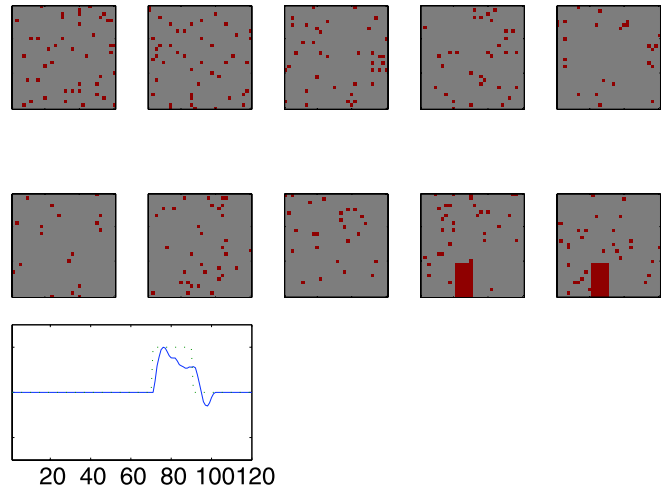

Component 4
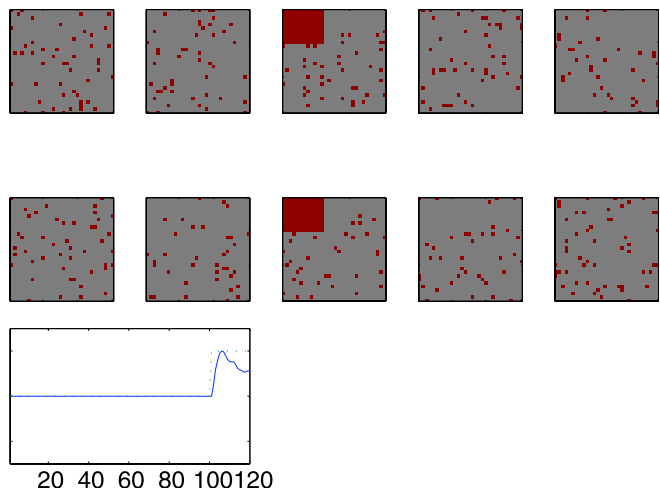

Component 1
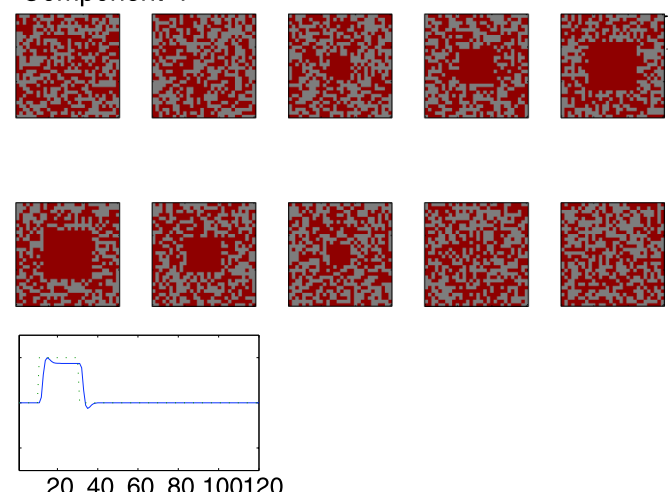

Component 2
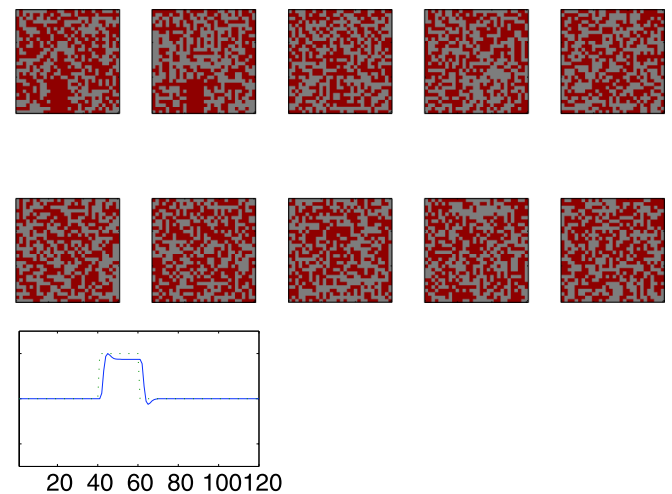

Component 3
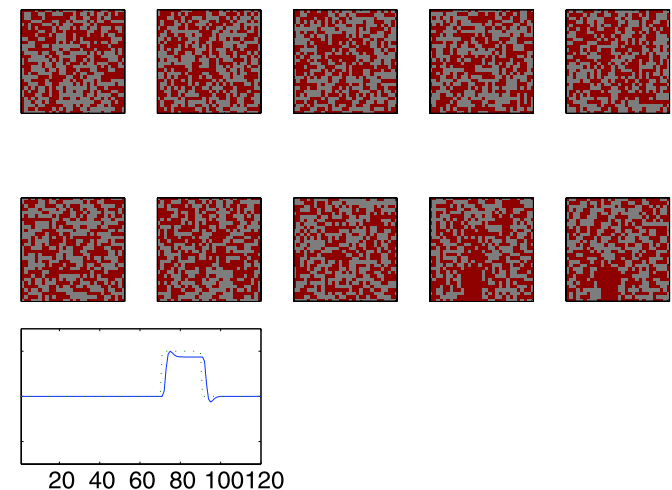

Component 4
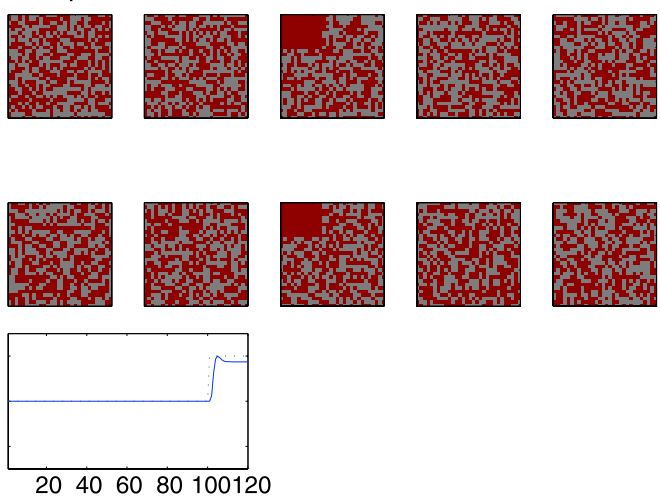

Figure 4. Comparison of the results from the proposed adaptive SPM approach (the left column) and SPM (the right column). 


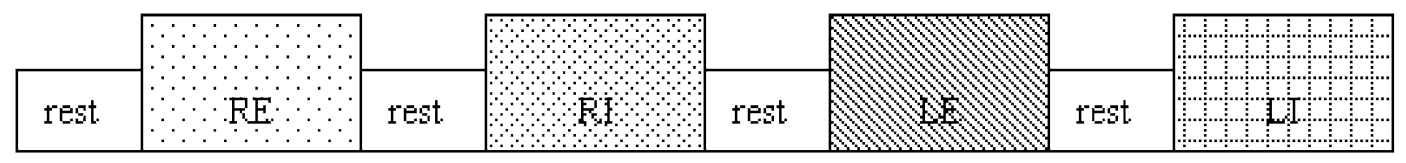

Figure 5. The experimental design used in acquiring the fMRI data. RE: EG Right-hand; RI: IG Right-hand; LE: EG Left-hand; LI: IG Left-hand. Each rest block took 30 seconds (10 scans when TR $=3$ seconds). Each activation block took 60 seconds (20 scans).

indicates higher intensity. Both methods demonstrate the classic brain activation patterns during hand movement as mentioned in Section 4.1. But our method shows dominantly higher intensity and less noise for each component. In addition, the subject is supposed to use more supplementary motor area (SMA, the third column) and less cerebellum (the first column) during the IG movement trial. This can be illustrated by the right-hand internally guided (RI) and left-hand internally guided (LI) rows, where the SMA with bright red color shown in the third slice is more profound than the cerebellum in the first slice (small red area).

Figure 7 presents the time component estimated by both methods. The solid line in each plot is the estimated time component and the dotted line is the stimulus sequence, where zero means "rest" and one means "activation". Because all the four components are assumed to share the same shape of the cerebral hemodynamic response, we only plot one time component here for each method. The time component estimated by our method has a hemodynamic shape that is much closer to reality.

\section{DISCUSSION}

In this paper, we introduced an adaptive SPM method to estimate the time components related to the fMRI experiment stimulus nonparametrically, which led to better detection of brain areas activated by the stimuli. The motivation was based on the fact that the detection power of SPM is constrained by usually predefined HRF. The idea of our method was to start from a design matrix $\mathbf{X}$ modeled by the canonical HRF followed with a penalized smoothing technique to refine the time component $(\mathbf{X})$ and spatial component in an iterative way. We used the timing information of the cerebral blood flow offered by the initial design matrix and adjusted the shape of the BOLD hemodynamic response of the activated voxels to be closer to the reality. We have illustrated the performance of our method through a comparative study on both simulated data and a real fMRI data set. Our method yielded better results in both cases. In addition, the procedure was easy to implement and the computation was fast.

There are, however, several unsettled issues which will now be described.

1. A crucial assumption employed in our development is the notion of the linear time-invariant system, or the convolution of the stimulus and the HRF. The departure of this assumption can have a moderate impact on our method, as well as many of the currently available methods. It has been reported that certain areas of the brain will exhibit some form of non-linearity, and it is certainly a very interesting problem on how to revise our approach to address this issue.

2. In addition to SPM5, we have also examined SPM8 carefully for the currently available options in selecting the HRF (an option of SPM), there is virtually no difference between the two versions. One useful option offered by SPM is to employ the finite impulse response model (FIR) approach in estimating the HRF. This is a less restricted approach as the parameters are not confined to a parametric function such as the canonical HRF based on the two-gamma model. The estimation is still carried out in two steps: estimate the HRF which is in turn being used to make an inference (by estimating the error covariance structure) about the fMRI activation. FSL [16] and AFNI [2] offer the same option. Both SPM and FSL are based on signal processing techniques [7], while AFNI is based on the regression method. Our procedure should be able to adapt itself to the FIR approach, and we will report this in the near future.

3. It will be interesting to compare our procedure when it is adapted to the spatio-temporal regularization method [17], the nonparametric method based on orthonormal causal Laguerre polynomials [14], the wavelet approach [12], the spline method [19], and the coherence based method [1].

4. This paper provides a method that estimates the design matrix used in SPM. The subsequent statistical inference problem is largely open. Presumably, one can do statistical inference of the parameters conditional on the estimated design matrix using available inference procedures for the general linear models [4]. However, the conditional inference may underestimate the uncertainty due to ignorance of the variability for estimating the design matrix. The magnitude of the underestimation is subject to future investigation. Another inference approach that deserves further study is the bootstrap method that randomly re-samples the residuals from our adaptive general linear model.

5. The above inference is related to the asymptotic properties of our convoluted estimator. It is conceivable that 


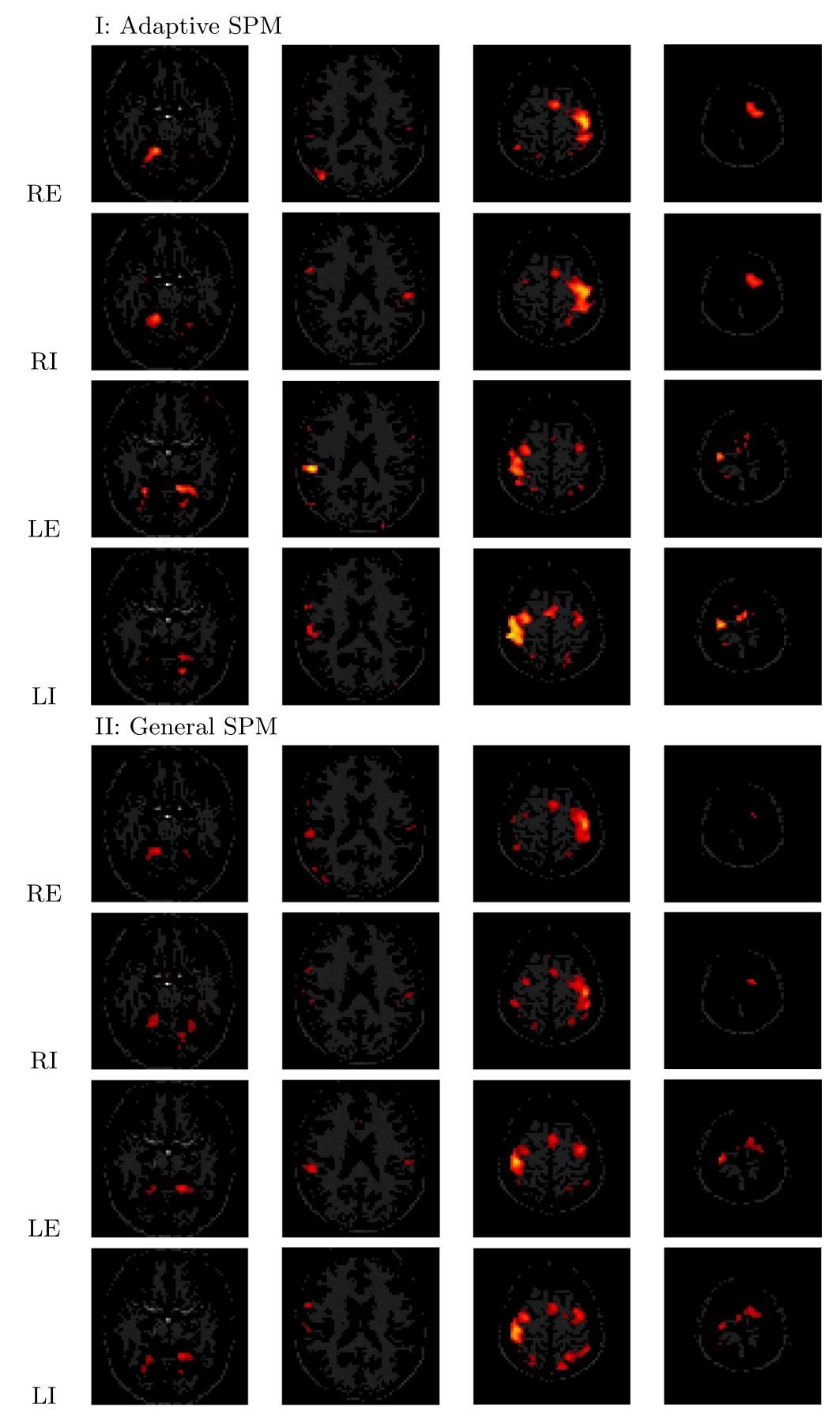

Figure 6. Brain regions activated by the four finger movements detected by two methods. Panel (I): Adaptive SPM, EG right-hand (first row), IG right-hand (second row), EG left-hand (third row) and IG left-hand (fourth row); Panel (II): SPM, EG right-hand (first row), IG right-hand (second row), EG left-hand (third row) and IG left-hand (fourth row). Within each row, the first slice shows cerebellum, the second slice shows basal ganglia, the third slice contains both primary motor cortex (PMC) and supplementary motor area (SMA), and the fourth slice shows the PMC. Red areas illustrate the activated voxels. Brighter color indicates higher intensity. 


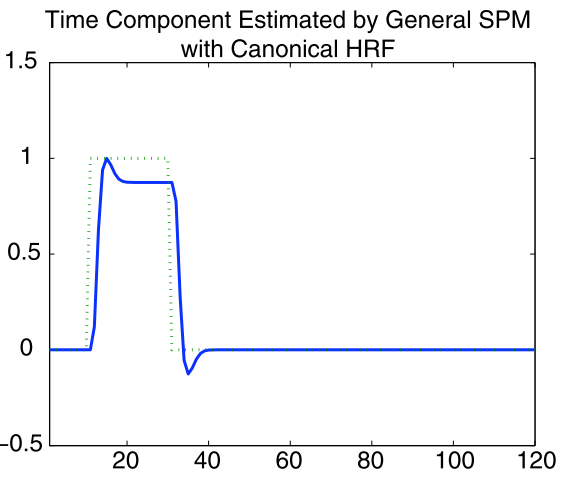

Figure 7. The time components (solid lines) estimated by two methods with the stimulus sequence (dotted lines) overlayed. Left: Adaptive SPM; Right: SPM with canonical HRF.

under some regularity conditions on the covariance of the noise series and the smoothness of HRF, optimal rates of convergence of the estimator can be established. This will not be too formidable for random noise models, but it may be challenging for temporally correlated noise.

\section{ACKNOWLEDGEMENTS}

We are grateful to Xuemei Huang, Mechelle Lewis and Drew Smith for making the fMRI data available for this project and their insightful comments on the physiology of our brains. We also thank the referees for their insightful and constructive comments that greatly improve the readability of the paper.

Received 6 August 2009

\section{REFERENCES}

[1] P. Bai, Y. Truong, and X. Huang, Nonparametric estimation of hemodynamic response function: A frequency domain approach, IMS Lecture Notes-Monograph Series. Optimality: The Third Erich L. Lehmann Symposium 57 (2009), 190-215.

[2] R. W. Cox, Afni: Software for analysis and visualization of functional magnetic resonance neuroimages, Computers and Biomedical Research 29 (1996), 162-173.

[3] K. J. Friston, J. Ashburner, S. J. Kiebel, T. E. Nichols, and W. D. Penny (eds.), Statistical parametric mapping: The analysis of functional brain images, Academic Press, 2007.

[4] K. J. Friston, A. P. Holmes, K. J. Worsley, J. P. Poline, C. D. Frith, and R. S. J. Frackowiak, Statistical parametric maps in functional imaging: a general linear approach, Human Brain Mapping 2 (1995), 189-210.

[5] C. R. Genovese, A Bayesian Time-Course Model for Functional Magnetic Resonance Imaging Data (with discussion), Journal of the American Statistical Association 95 (2000), no. 451, 691-703.

[6] G. H. Glover, Deconvolution of impulse response in event-related fmri, NeuroImage 9 (1999), 416-429.

[7] C. Goutte, F. A. Nielsen, and L. K. Hansen, Modeling the haemodynamic response in fMRI using smooth FIR filters, IEEE Transactions on Medical Imaging 19 (2000), 1188-1201.

[8] T. Hastie, R. Tibshirani, and J. H. Friedman, The elements of statistical learning, Springer, 2003.
[9] D. Hu, L. R. Yan, Y. D. Liu, Z. T. Zhou, K. J. Friston, C. L. Tan, and D. X. Wu, Unified SPM-ICA for fMRI analysis, NeuroImage 25 (2005), 746-755.

[10] O. Josephs, R. Turner, and K. Friston, Event-related fmri, Human Brain Mapping 5 (1997), 243-248.

[11] F. Kherif, J-B. Poline, G. Flandin, H. Benali, O. Simon, S. Dehaene, and K. J. Worsley, Multivariate model specification for fmri data, NeuroImage 16 (2002), 1068-1083.

[12] C. Long, E. N. Brown, D. Manoach, and V. Solo, Spatiotemporal wavelet analysis for functional mri, NeuroImage 23 (2004), 505516.

[13] W. S. Rayens and A. H. Andersen, Multivariate analysis of fMRI data by oriented partial least squares, Magnetic Resonance Imaging 24 (2006), 953-958.

[14] S. Saha, C.J. Long, E. Brown, E. Aminoff, M. Bar, and V. Solo, Hemodynamic transfer function estimation with laguerre polynomials and confidence intervals construction, from functional magnetic resonance imaging (fmri) data, Proc IEEE ICASSP III (2004), 109-112.

[15] S. Sen, A. Kawaguchi, Y. K. Truong, M. M. Lewis, and X. Huang, Dynamic changes in cerebello-thalamo-cortical motor circuitry during progression of parkinson's disease, Neuroscience 166 (2010), 712-19.

[16] S. M. Smith, M. Jenkinson, M. W. Woolrich, C. F. Beckmann, T. E. J. Behrens, H. Johansen-Berg, P. R. Bannister, M. De Luca, I. Drobnjak, D. E. Flitney, R. Niazy, J. Saunders, J. Vickers, Y. Zhang, N. De Stefano, J. M. Brady, and P. M. Matthews, Advances in functional and structural $\mathrm{mr}$ image analysis and $\mathrm{im}-$ plementation as fsl, NeuroImage 23(S1) (2004), 208-219.

[17] V. Solo, P. Purdon, R. Weisskoff, and E. Brown, A signal estimation approach to functional mri, IEEE Transactions on Medical Imaging 20 (2001), 26-35.

[18] C. M. Zhang, and T. Yu, Semiparametric detection of significant activation for brain fmri, Ann. Statist. 36 (2008), 1693-1725. MR2435453

[19] C. M. Zhang, Y. Jiang, and T. Yu, A comparative study of onelevel and two-level semiparametric estimation of hemodynamic response function for fmri data, Statistics in Medicine 26 (2007), 3845-3861. MR2395874

Ping Bai

PayPal Inc.

2211 N First Street

San Jose, CA 95131, USA

E-mail address: pingbai@gmail.com 
Haipeng Shen

Department of Statistics and Operations Research University of North Carolina at Chapel Hill Chapel Hill, NC 27599, USA

E-mail address: haipeng@email.unc.edu

Jianhua Z. Huang

Department of Statistics

Texas A\&M University

College Station, TX 77843, USA

E-mail address: jianhua@stat.tamu.edu
Young K. Truong

Department of Biostatistics

Department of Neurology

University of North Carolina at Chapel Hill

Chapel Hill, NC 27599, USA

E-mail address: truong@bios .unc.edu 